\title{
Phase-resonance Exploitation in Full-Metal 3D Periodic Structures for Single- and Multi- Wideband Applications
}

This paper was downloaded from TechRxiv (https://www.techrxiv.org).

\section{LICENSE}

CC BY 4.0

SUBMISSION DATE / POSTED DATE

23-12-2021 / 28-12-2021

\section{CITATION}

Molero Jiménez, Carlos (2021): Phase-resonance Exploitation in Full-Metal 3D Periodic Structures for Singleand Multi- Wideband Applications. TechRxiv. Preprint. https://doi.org/10.36227/techrxiv.17439956.v1

$\mathrm{DOI}$

10.36227/techrxiv.17439956.v1 


\title{
Phase-resonance Exploitation in Full-Metal 3D Periodic Structures for Single- and Multi- Wideband Applications
}

\author{
M. A. Balmaseda-Márquez, S. Moreno, P. H. Zapata, and C. Molero, Member, IEEE,
}

\begin{abstract}
This paper presents a versatile full-metal 3D periodic structure based on square waveguides with non-closed resonators perforated on their walls. The complex unit-cell architecture is modelled via accurate equivalent circuits, previously characterized. The circuit model predicts the excitation of phase resonance, which will be used to optimize and design different functionalities, such as polarisation converters or absorption.
\end{abstract}

Index Terms-Polarisation converters, 3D full-metal structures, phase resonance.

\section{INTRODUCTION}

$\mathbf{T}$ HE discovery of exotic electromagnetic phenomena have always attracted a big amount of researchers due to several reasons, such as the scientific curiosity and the emergence of novel applications. It is for instance noteworthy the interest paid on the extraordinary-transmission phenomenon [1] and the promising fields of applications that were theorized at the beginning of the current century [2].

The study of phase resonance, originally conceived as the mutual resonance between adjacent slits in compound gratings [3], has theoretically been described but no apparent applicability seems to exist. Experimentally it is manifested in a similar way as an electric resonance [4], by means of a full transmission dip in transmission structures [5], [6], or by $180^{\circ}$-phase in reflection mode [7]. From the theoretical point of view, simple interpretations via modelling based on equivalent circuits were successfully realized [8], [9], clarifying some key aspects and generalizing the scenarios where phase resonance can appear. For example, circuit topologies including transmission lines connected in series are prone to exhibit phase resonance [8].

Not many applications profiting from the properties of phase resonance can be found. Dr. Skigin et al. reported an interesting associated phenomenon called superdirectivity in finite grooved gratings [7]. It is an ultra directive beam, manifested at the phase-resonance frequency and of narrow-band nature. Phase resonance has also been employed for band isolation, especially in 3D multi-band designs. In these contexts it appears as a sudden reflection peak in the intermediate region between two operation bands, reducing interferences. Some prototype examples are reported in [10], [11] presenting 3D periodic

Manuscript received XX - XX - XXXX; revised XX - XX- XXXX; accepted $\mathrm{XX}-\mathrm{XX}-\mathrm{XXXX}$. This work was supported by the BBVA foundation through a project supported by a 2021 Leonardo Grant for Researchers and Cultural Creators, BBVA Foundation. The BBVA Foundation accepts no responsibility for the opinions, statements and contents included in the project and/or the results thereof, which are entirely the responsibility of the authors structures based on PCB-models and coaxial waveguide-cells respectively, or in fully-metallic 3D architectures [12].

The use of 3D periodic-structures and metasurfaces is a research field in expansion thanks to, among other things, their inherent versatility [13], [14] and the emerging development of 3D-printing techniques [15], [16]. Special attention deserves full-metal devices [12], [17], [18], highly demanded in spacial applications [19]. These configurations, based on square waveguides with resonant perforations on the walls, have been successfully tested in the laboratory, but generally suffer from narrow-band operation. Wideband-operation response demands a full redefinition of the perforation shapes and geometries.

This paper presents an enhancement of the unit cell in [12], including two fundamental modifications. A first modification regards the inclusion of a ground plane, enabling reflectionmode operation only. A second modification concerns the resonators shape, that will include non-closed geometries. In particular short-terminated slits (in contrast to dog-bones or

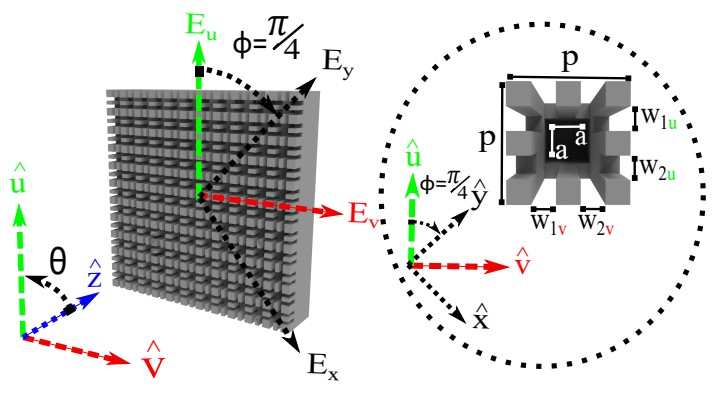

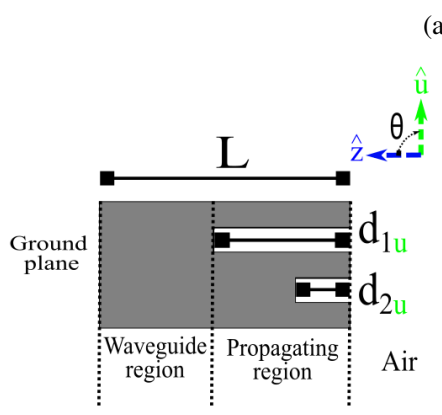

(b) a)

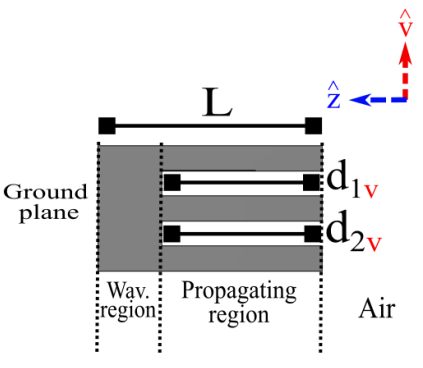

(c)

Fig. 1. 11x11-array in perspective and unit cell with the corresponding structure parameters. (a) Complete structure and front view of the cell, (b) lateral view, parallel to the $\hat{\mathbf{u}}$-component, (c) Top view, parallel to the $\hat{\mathbf{v}}$ component. 
folded slots are in [12]), giving rise to a sort of compoundgrating version, as depicted in Fig. 1(a). Phase resonance is excitable in these cells, and its manifestation in the form of electric resonance will be applied to attain several functionalities. In addition, the design and optimization will be realized via circuit models, where the ideas and experiences previously reported in [20], [21] have succesfully been applied.

The paper is organized as follows: Section II where the circuit topology is proposed and tested, leading to the optimization of two cell geometries for two different functionalities; and Section III showing the versatility of the cell employed for the conception of rotators and absorbers.

\section{Cell Defintion And Characterization}

The unit-cell type presented in this paper is a regular square waveguide with slits perforated on the walls. One of the fundamental differences with the cell in [12] is the use of non-closed resonators in the form of slits, which decrease the inherent Qfactor of the whole structure. Fig. 1 illustrates the front, top, and lateral views of the cell. The slits do not reach down to the end of the cell, so that two different sections can be identified: on the one hand, the section containing the slits, corresponding to a propagating region. The number of slits is not restricted to two, i. e., the cell can support as many slits as needed, provided that they fit according to the cell dimensions. On the other hand, the waveguide region, where the dominant field is described by the $\mathrm{TE}_{10}$-mode. The contribution of this mode will generally be reactive, since the dimensions of the unit cell and operation frequency will be established to ensure no modal excitation (cutoff region). Furthermore, the waveguide region is terminated in ground plane, forcing full-reflection operation and constituting a second fundamental difference with the cell in [12]. The structure is illuminated by an external plane wave. Thanks to the 3D character of the cell, independent control of different field components can be obtained. In this sense, lateral perforations (see Fig. 1(b)) manipulate the $\hat{\mathbf{u}}$ component of the incident wave only, whereas top/bottom perforations (see Fig. 1(c)) control the corresponding $\hat{\mathbf{v}}$ component. This allows to split the problem into two independent sub-problems, addressed hereafter in terms of two individual equivalent circuits.

\section{A. Circuit-model characterization.}

A proper circuit topology to replicate the functionality of the cell is shown in Fig. 2. The transmission lines (TLs) connected in series $\left(Z_{1}, Z_{2}\right)$ refer to the different propagation channels associated with each individual slit. We can identify the following parameters: $Z_{0}$ being the characteristic impedance (CI) of a TL representing the incident and reflected waves in free space; $C_{i}$ the individual capacitance describing the fringe fields at each of the slit apertures; $Z_{i}$ and $d_{i}$ describing CIs and lengths of the TLs associated with each of the slits in the propagation region respectively, and $Z_{10}^{\mathrm{TE}}$ being the $\mathrm{CIs}$ for the TLs describing the decay of the $\mathrm{TE}_{10}$ in the waveguide region. This TL is short-circuited in order to include the effect of the ground plane. The transformers $N_{i}$ account for the coupling between the modes on the propagating region and the $\mathrm{TE}_{10}$ mode, as it is similary described in [20]. It is worth remarking that the number of TLs connected in series in the propagating region is related to the number of slits on the cell walls. If more than two slits are considered, more than two TLs must be included.

The value of the parameters previously described depends on the cell dimensions. In principle, the impedance in free space, assuming normal incidence, is taken into account as $Z_{0}=\eta_{0}=376.73 \Omega$. Similarly, the impedance associated with the $\mathrm{TE}_{10}$ mode is that of a regular waveguide [22]:

$$
Z_{10}^{\mathrm{TE}}=\frac{c \sqrt{(\omega / c)^{2}-(\pi / a)^{2}}}{\eta_{0} \omega} \Omega,
$$

with $\omega$ being the angular frequency and $c$ the speed of light. Finally, the TL lengths are those of the slits: $d_{i}$ in the case of the slits and $L-d_{i}$ for the TE lines. The remaining parameters $Z_{i}, C$ and $N_{i}$ must be evaluated numerically. For this, we first consider a waveguide model with a single slit and its corresponding equivalent circuit. They have been inserted in Fig. 3(a). We have arbitrarily chosen the $\hat{\mathbf{u}}$-component as the incidence field (the conclusions from this component are identical for the orthogonal one). The obtaining of the parameters has been carried out with the help of the commercial software CST Studio Suite. A unit cell model having fixed $L$ and $d$ is employed. It is worth remarking that the parameters $L$ and $d$ do not affect the numerical calculation. Thus, the $S_{11}$ is computed in CST for different $w / p$. A fixed $w / p$ value leads to a fixed set of values of $Z, C, N$, achieved after some mathematical calculations. It is assumed that $Z, C, N$ are not very sensitive to $a / p$ in scenarios where $a / p \geq 0.6$.

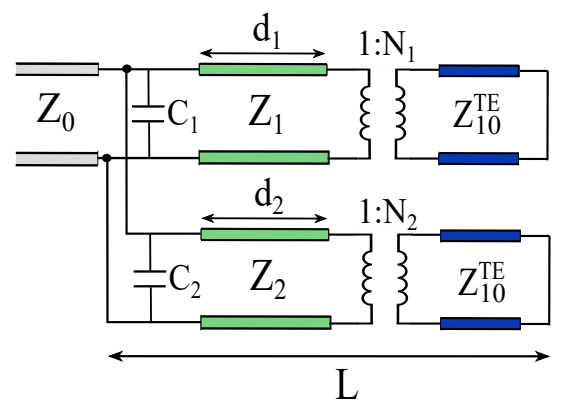

Fig. 2. Equivalent circuit of the proposed unit cell with double slit.

Fig. 3(a) and Fig. 3(b) show the evolution of this set of parameters with respect to $w / p$. As expected, the capacitance decreases as the slit width increases. The inverse behavior is otherwise observed for the CI and the transformer, denoting a sort of logarithmic trend. The plots in Fig. 3 will be highly useful to construct the cell with more than one slit, and it will be employed to design and optimize the cell for wideband functionalities.

\section{B. Single slit, double-symmetric slit, double-asymmetric slit.}

Once the circuit elements have been characterised, we will test the circuit validity by plotting the phase of the reflection coefficient for three different cell configurations: a first case consisting on a cell with a single slit perforated on the wall; 


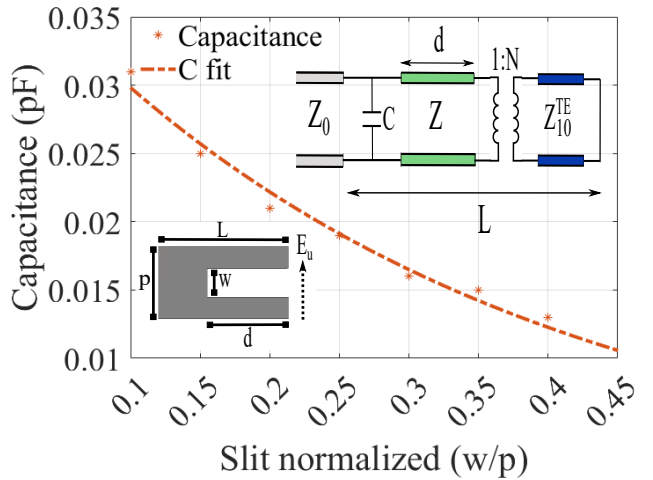

(a)

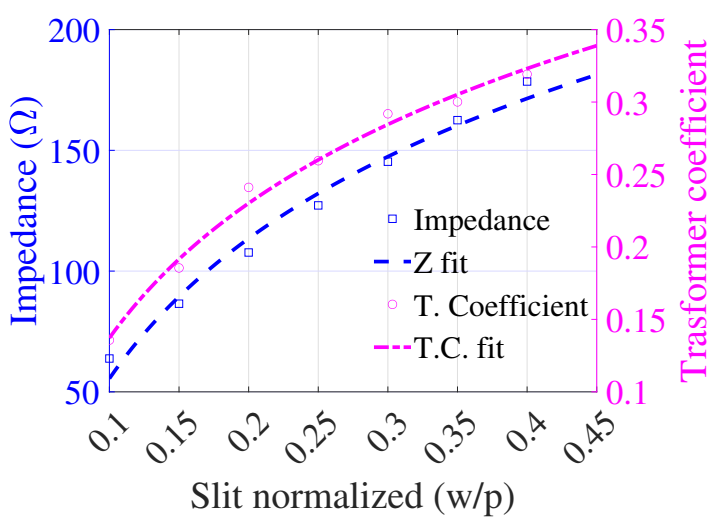

(b)

Fig. 3. Impedance, capacitance and transformer coefficient vs normalized slit: (a) Capacitance (b) Impedance and transformer coefficient. Fixed parameters: $L=20 \mathrm{~mm}$ and $d=14 \mathrm{~mm}$.

a second case considering two identical slits; and a third case concerning two slits with different lengths. The evaluation will be done for the incident components $\hat{\mathbf{u}}$ and the conclusions will be extended to the orthogonal one. The value of the circuit elements has been extracted from the curves represented in Fig. 3. In the double-slit cases under study, $Z_{i}, C_{i}, N_{i}$ of each individual TL have also been taken from Fig. 3 as if the TL were isolated. This constitutes an approximation in our model. However, as shown in Fig. 4, the agreement between curves extracted from CST and the equivalent circuits is excellent. It is worth noting that no major difference exists between the single case and the double-slit case when they are identical (blue and black curves). The phase evolution is the typical of shorted TLs in both cases: it starts from $180^{\circ}$, crosses the $0^{\circ}$ when the wavelength is approximately $\lambda / 4$ and comes back to $180^{\circ}$ at frequencies close to $\lambda / 2$ (notice that the presence of the $\mathrm{TE}_{10}$ line prevents the coincidence of the resonances at $\lambda / 4$ and $\lambda / 2$, respectively). However, the most interesting result is that where the slits are different (red curves). The geometrical parameters of the cell are identical to those of the second case excepting for the length of one of the slits, that has been shortened. This small variation on the geometry induces the appearance of a sudden resonance around $6 \mathrm{GHz}$. It is manifested by a $180^{\circ}$ phase value, and its presence is owing to the inner interaction between both slits. This resonance is the

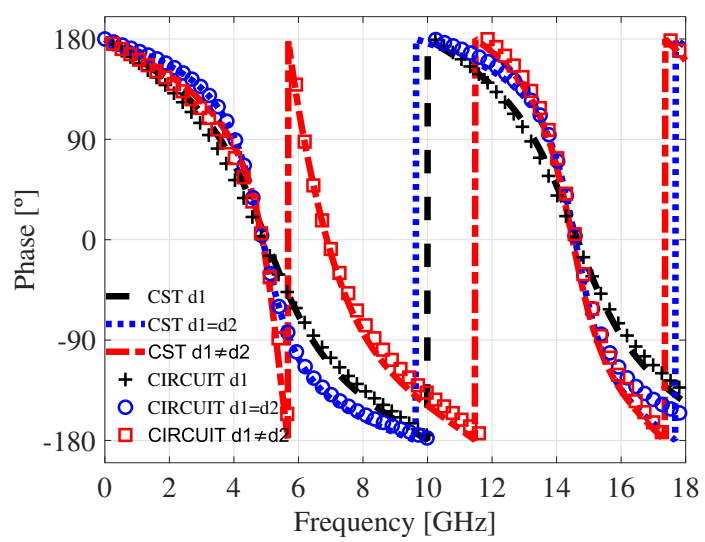

Fig. 4. Phase of the reflection coefficient versus frequency for three different cell configurations. Common structure parameters: $p=10 \mathrm{~mm}, L=20 \mathrm{~mm}$. Parameters in single slit cell: $d=14.3 \mathrm{~mm}, w=2 \mathrm{~mm}$. Parameters in identical double-slit case: $d_{1}=d 2=14.3 \mathrm{~mm}, w_{1}=w_{2}=2 \mathrm{~mm}$. Parameters in different double-slit case: $d_{1}=14.3 \mathrm{~mm}, d_{2}=9.8 \mathrm{~mm}$, $w_{1}=w_{2}=2 \mathrm{~mm}$. Circuit parameters numerically obtained for single slits: $Z=105 \Omega, C=0.021 \mathrm{pF}, N=0.25$. Circuit parameters numerically obtained for double-slit: $Z_{1}=Z_{2}=105 \Omega, C_{1}=C_{2}=0.021 \mathrm{pF}$, $N_{1}=N_{2}=0.25$.

so-called phase resonance. From the circuit point of view it is interpreted as a series resonance between two different reactive elements. In spite of the simplicity of the equivalent circuit and the topology, the phase-resonance behavior is well reproduced. This gives consistency to the proposal, becoming an efficient design tool for applications involving phase control.

\section{Accurate design of unit cells via the circuit model.}

This section is intended to explore the range of applicability of the cell. In particular, to design a wideband rotator and a wideband dual-pol polarizer. For this, we will rely on the double-slit cells described on the previous section. We first begin by the design of a field rotator. The structure is illuminated by a plane wave impinging normally and $\hat{\mathbf{y}}$-polarized electric field (according to the frame of coordinates of Fig. 1(a)). This field can be decomposed into $\hat{\mathbf{u}}$ - and $\hat{\mathbf{v}}$-components, splitting the problem into two independent subproblems. The optimization of each component has individually been addressed via its corresponding circuit model. An efficient field rotation (from $\hat{\mathbf{y}}$ to $\hat{\mathbf{x}}$ ) is achieved when the phases of $\hat{\mathbf{v}}$ - and $\hat{\mathbf{u}}$-components are shifted by $180^{\circ}$ [23]. This can be realized just by inducing the correct resonances on the slits. Fig. 5(a) shows the phase of the reflection coefficient for both components obtained by specific circuit models (see the caption for more information). As shown, the $\hat{\mathbf{u}}$-component $\left(\mathrm{E}_{\mathrm{u}}\right.$ in the figure) crosses $0^{\circ}$-phase at $10 \mathrm{GHz}$, coinciding with $180^{\circ}$-phase of the $\hat{\mathbf{v}}$-component $\left(E_{v}\right)$. This ensures a wideband $180^{\circ}$ phase-shift. The circuital optimization leads to identical-slits topologies in both cases $\left(d_{1 \mathrm{u}}=d_{2 \mathrm{u}}=6.5 \mathrm{~mm}, d_{1 \mathrm{v}}=d_{2 \mathrm{v}}=14.5 \mathrm{~mm}\right)$. The agreement with CST is excellent as it can be checked. Fig. 5(b) illustrates the effective rotation of the electric field. These values are accepted since the amplitude of the incident wave component $\mathrm{E}_{\mathrm{y}}$ decays below $-10 \mathrm{~dB}$, transmitting the power the other component $\mathrm{E}_{\mathrm{x}}$ for a bandwidth of almost $93 \%$ (from $5.3 \mathrm{GHz}$ to $14.4 \mathrm{GHz}$ ). 


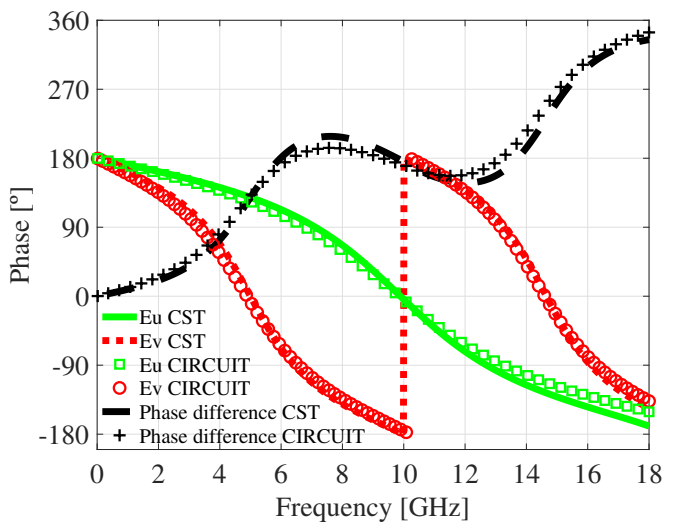

(a)

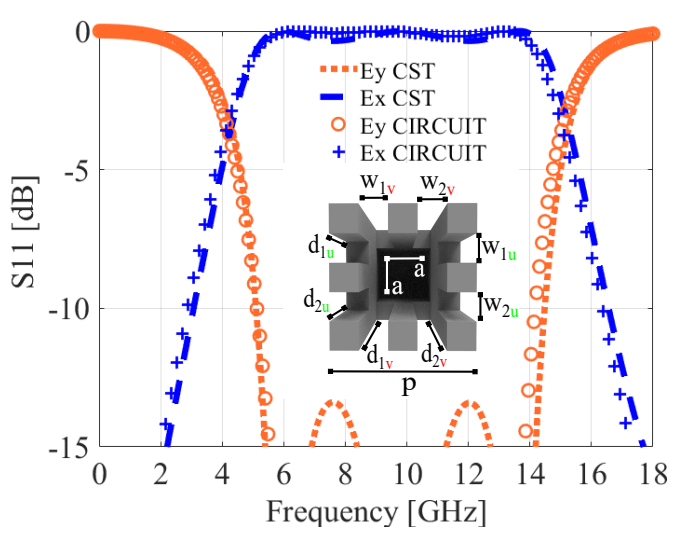

(b)

Fig. 5. Design of a field rotator. (a): Phase of the reflection coefficient of both $\mathrm{E}_{\mathrm{u}}$ and $\mathrm{E}_{\mathrm{v}}$. (b): Reflection coefficient for the co-polarized component $\mathrm{E}_{\mathrm{y}}$ and the cross-polarized one $\mathrm{E}_{\mathrm{x}}$. Geometrical parameters: $p=10 \mathrm{~mm}$, $a=6 \mathrm{~mm}, L=20 \mathrm{~mm}, d_{1 \mathrm{u}}=d_{2 \mathrm{u}}=6.5 \mathrm{~mm}, d_{1 \mathrm{v}}=d_{2 \mathrm{v}}=14.3 \mathrm{~mm}$, $w_{1 \mathrm{u}}=w_{2 \mathrm{u}}=w_{1 \mathrm{v}}=w_{2 \mathrm{v}}=2 \mathrm{~mm}$. Circuit parameters: $C_{1}=C_{2}=$ $0.021 \mathrm{pF}, Z_{1}=Z_{2}=105 \Omega, N_{1}=N_{2}=0.25$.

A second example of application consists of a dual-band polarizer with polarisation inversion. For this case, we will take advantage of the phase resonance. Again, the optimization will be carried out by the equivalent circuit. The cell receives the incidence of a plane wave normally oriented and whose electric-field vector is polarized along $\hat{\mathbf{y}}$. The problem is again decomposed into $\hat{\mathbf{v}}$ - and $\hat{\mathbf{u}}$-subproblems. The optimization process results in identical slits on the top/bottom walls $\left(d_{1 \mathrm{v}}=d_{2 \mathrm{v}}=14.3 \mathrm{~mm}\right)$, but nevertheless leads to different slits $\left(d_{1 \mathrm{u}}=10.9 \mathrm{~mm}\right.$ and $\left.d_{2 \mathrm{u}}=4.3 \mathrm{~mm}\right)$ for those on lateral walls. The phase evolution of both components is plotted in Fig. 6(a). Since $d_{1 \mathrm{v}}, d_{2 \mathrm{v}}$ are larger than $d_{1 \mathrm{u}}, d_{2 \mathrm{u}}$, the phase associated with $\mathrm{E}_{\mathrm{v}}$ is advanced with respect to the phase of $\mathrm{E}_{\mathrm{u}}$ at lower frequencies. This tendency is kept up to $7.5 \mathrm{GHz}$, frequency at which it gets reversed. Before the change of tendency, a $90^{\circ}$ phase-shift is induced from 4 to $6 \mathrm{GHz}$. The change of tendency is forced by the appearance of a phase resonance at $9 \mathrm{GHz}$, provoking the phase delay of $E_{v}$ with respect $E_{u}$. In addition, the delay has been optimized to force a wideband $-90^{\circ}$ phase-shift beyond $10 \mathrm{GHz}$. Fig. 6(b) represents the reflection coefficient in terms of the circular

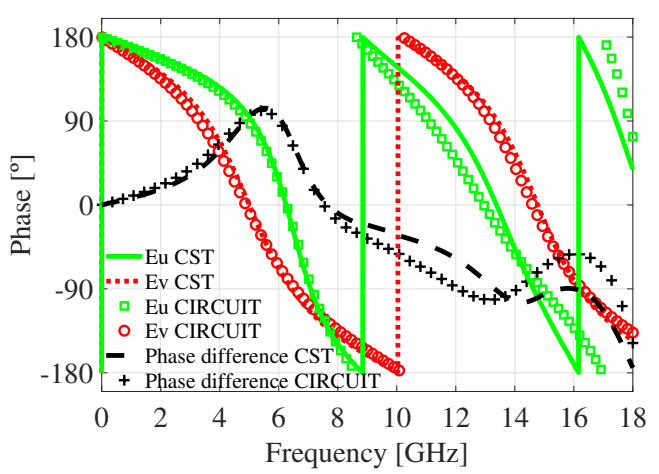

(a)

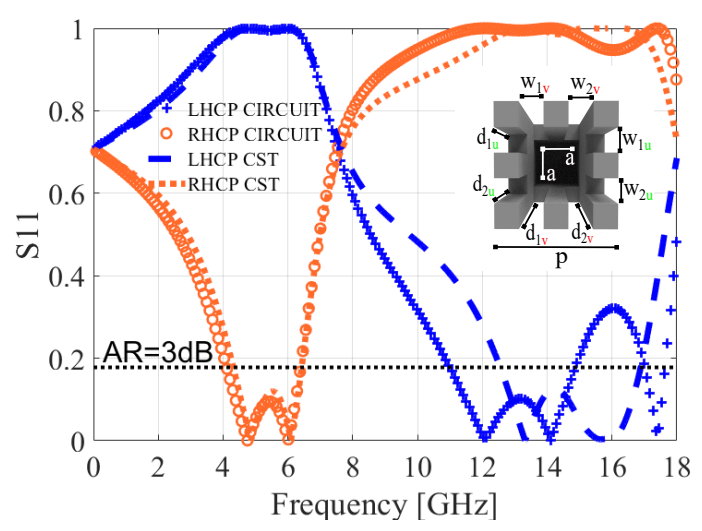

(b)

Fig. 6. Design of a dual-band polarizer. (a): Phase of the reflection coefficient of both $\mathrm{E}_{\mathrm{u}}$ and $\mathrm{E}_{\mathrm{v}}$. (b): Reflection coefficient of the LHCP- and RHCPcomponents. Geometrical parameters: $p=10 \mathrm{~mm}, a=6 \mathrm{~mm}, L=20 \mathrm{~mm}$, $d_{1 \mathrm{u}}=10.9 \mathrm{~mm}, d_{2 \mathrm{u}}=4.3 \mathrm{~mm}, d_{1 \mathrm{v}}=d_{2 \mathrm{v}}=14.3 \mathrm{~mm}, w_{1 \mathrm{u}}=$ $w_{2 \mathrm{u}}=w_{1 \mathrm{v}}=w_{2 \mathrm{v}}=2 \mathrm{~mm}$. Circuit parameters: $C_{1}=C_{2}=0.021 \mathrm{pF}$, $Z_{1}=Z_{2}=105 \Omega, N_{1}=N_{2}=0.25$.

base:

$$
\begin{aligned}
& \mathbf{S}_{11}^{\mathrm{RHCP}}=\frac{1}{2}\left(S_{11}^{\mathrm{v}}+j S_{11}^{\mathrm{u}}\right) . \\
& \mathbf{S}_{11}^{\mathrm{LHCP}}=\frac{1}{2}\left(S_{11}^{\mathrm{v}}-j S_{11}^{\mathrm{u}}\right),
\end{aligned}
$$

where $S_{11}^{\mathrm{u} / \mathrm{v}}$ are the complex-valued reflection coefficients of $\hat{\mathbf{u}}$ and $\hat{\mathbf{v}}$ - components respectively. The polarisation inversion is easily visualized, having LHCP from 4.3 to $6.4 \mathrm{GHz}(40 \%)$ and RHCP from 12.6 to $16.9 \mathrm{GHz}(30 \%)$. The bandwidth is defined by a threshold imposed to the cross-component. This threshold, set at $S_{11}=0.19$, indicates the limit below which the axial-ratio parameter is under $3 \mathrm{~dB}$. It is noteworthy the slight deterioration of the results provided by the circuit model at the higher band, basically owing to the $\hat{\mathbf{u}}$-component. This disagreement shows the limitation of the model for higher frequencies and especially when the slits are different. Otherwise the model is still useful for optimization and design since it predicts the band position and bandwidth. A quick optimization is CST was eventually necessary to achieve a slightly better performance. 


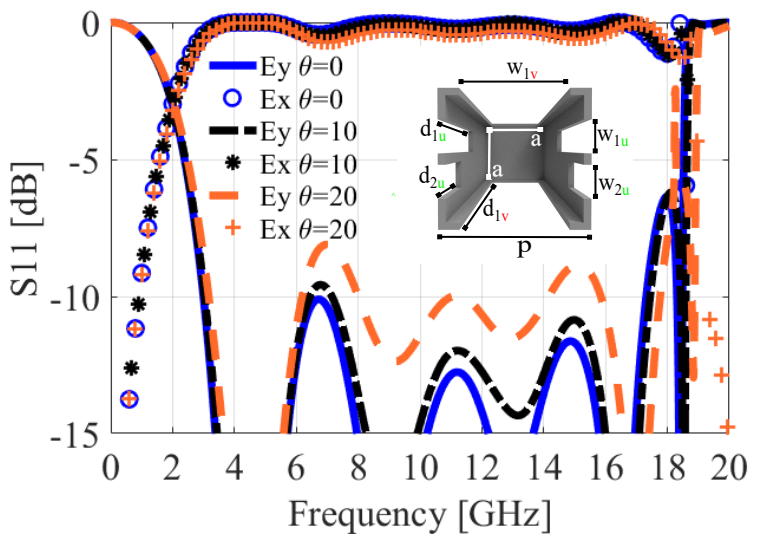

Fig. 7. Reflection coefficient for the co-polarized component $\mathrm{E}_{\mathrm{y}}$ and the cross-polarized one $\mathrm{E}_{\mathrm{X}}$. Geometrical parameters: $p=10 \mathrm{~mm}, a=9 \mathrm{~mm}$, $L=30 \mathrm{~mm}, d_{1 \mathrm{u}}=13.9 \mathrm{~mm}, d_{2 \mathrm{u}}=6.3 \mathrm{~mm}, d_{1 \mathrm{v}}=30 \mathrm{~mm}, w_{1 \mathrm{u}}=w_{2 \mathrm{u}}=$ $2.4 \mathrm{~mm}, w_{1 \mathrm{v}}=7.2 \mathrm{~mm}$.

\section{VERSATILITY OF THE CELL}

This section is entirely dedicated to test the utility and versatility of the cell in different scenarios, as the perforation of large-width slits or the increase of the number of slits per wall. In particular, a larger number of slits increases the complexity of the phenomenology and the circuit-model representation, but also enhance the performance of several functionalities, gaining in robustness and bandwidth. Furthermore, absorbers in the role of dual-pol rasorbers [24] can easily be designed as it will be discussed in the following.

\section{A. Ultra-wideband rotator}

An ultra wideband rotator can be obtained by properly optimizing the cell under consideration. It has been conceived to operate from 3 to $18 \mathrm{GHz}$ covering a fractional bandwidth of $140 \%$. The circuit optimization has been done for normal incidence, though simulated results for oblique incidence $\theta \neq 0$ are also included. As shown in the inset of Fig. 7, the optimization has lead to a cell configuration where top/bottom walls have a single slit only, which has been enormously widened $\left(w_{\mathrm{v}}=7.2 \mathrm{~mm}, w_{\mathrm{v}} / p=0.72\right)$. The lateral walls are perforated with a pair of narrower slits of different dimensions $\left(w_{1 \mathrm{u}}=w_{2 \mathrm{u}}=2.4 \mathrm{~mm}, d_{1 \mathrm{u}}=13.9 \mathrm{~mm}, d_{2 \mathrm{u}}=6.3 \mathrm{~mm}\right)$. Since both slits are different, phase resonance is excited, taking an important role to reach the final performance. The result is presented in Fig. 7, where the fractional bandwidth of $140 \%$ can be appreciated. The figure focuses on the final results obtained by CST for normal and oblique incidence. As observed, a little degradation appears in the field rotation at $\theta=20^{\circ}$, though the performance can still be considered very good.

\section{B. Dual-pol rasorber}

A final case consists of the design of a rasorber, whose spectral response is characterized by a band sequence absorptionreflection-absorption for each incident polarisation [24]. Now, a five-slits-per-wall cell is used, as illustrated in Fig. 8(a).
The cell geometry is such that it reflects one of the incident polarisations at the same band where the orthogonal one is absorbed, and vice versa. This creates 2 different and independent reflection channels. With this in mind, it is necessary to introduce resistive materials in the cell. Good candidates are resistive layers (commercially available) with negligible thickness and characterized by a sheet-resistance value $R_{s}$. Fig. 8(b) and Fig. 8(c) show their dimensions and the way they are introduced in the cell. Circuitally, the presence of the resistive sheet is described by an additional resistance, as depicted in Fig. 8(a), whose value may be estimated as:

$$
R=R_{s} \frac{W_{c}}{L_{c}}
$$

with $L_{c}$ and $W_{c}$ being the chip dimensions. The circuit in Fig. 8(a) refers to slit where the chips are included, taken into account by an effective resistance $R$ parallel connected at a distance $m$ from the $T E_{10}$-TL. The Absorption coefficient of a design optimized for the rasorber is plotted in Fig. 8(a). Results from both TM and TE indcidence are included for different incidence angles. The chip distribution is such that they are included in external and middle slits, since they are the shortest and largest ones respectively. This invokes an absorption band at lower frequencies, associacted with the individual resonances of largest slit, and another at higher frequencies related to the individual resonances of shortest slits. A $R_{s}=25 \Omega / \mathrm{sq}$ was necessary to attain optimum absorption in both bands. The second and fourth slits control the position of the reflection channel, in the middle between the both absorption bands. Thanks to the independence at controlling $\hat{\mathbf{v}}$ - and $\hat{\mathbf{u}}$-components, absorption band of $\mathrm{E}_{\mathrm{u}}$ can coincide with the reflection band of $E_{\mathrm{v}}$ just by playing with the slit lengths and their resonances. In this case, phase resonance helps to isolate absorption from reflection bands for a same polarisation. Fig. 9 shows this behavior, which is kept up to larger incidence angles. The independent control for both field components makes it this actually interesting for radar applications.

\section{CONCLUSION}

The versatility of the unit cell has been shown in the present manuscript. The number of slits and their geometry determines the features of multiple functionalities, specially for multiband and wideband applications. The equivalent circuit has been demonstrated as a very useful tool for the design, besides its simplicity. Further steps consists on the fabrication by stereolithography and the experimental testing, expected in a few months.

\section{ACKNOWLEDGMENTS}

The authors acknowledge the support of the BBVA foundation for the funds associated to a project belonging to the program Leonardo Grants 2021 for researchers and cultural creators from the BBVA foundation. 


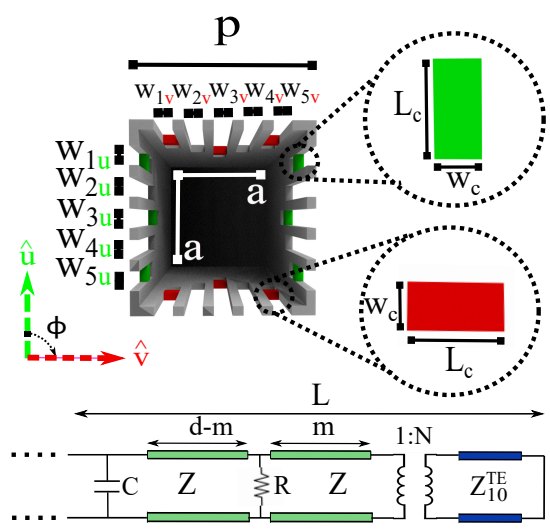

(a)

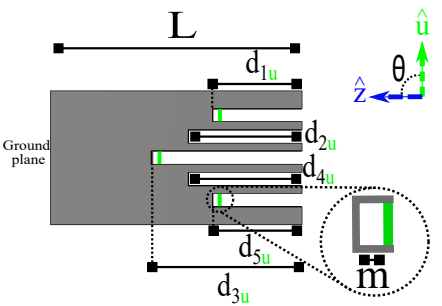

(b)

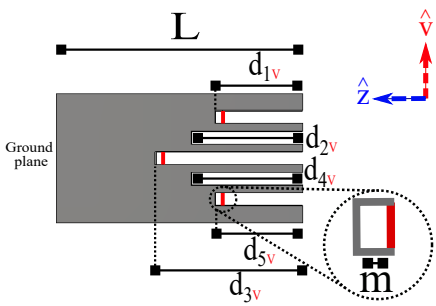

(c)
Fig. 8. Unit cell and corresponding parameters. Circuit model. (a) Front view of the cell. (b) Lateral view, parallel to the $\hat{\mathbf{u}}$-component. (c) Top view, parallel to the $\hat{\mathbf{v}}$-component.

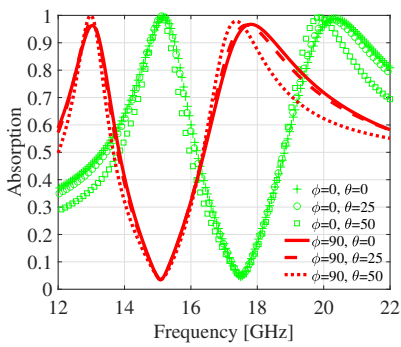

(a)

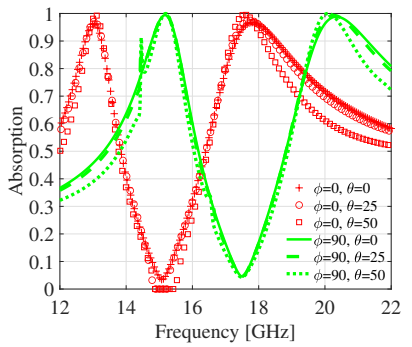

(b)
Fig. 9. (a): Absorption TE, (b): Absorption TM. Geometrical parameters: $p=5 \mathrm{~mm}, a=4.5 \mathrm{~mm}, L=10 \mathrm{~mm}, d_{1 \mathrm{u}}=d_{5 \mathrm{u}}=3.12 \mathrm{~mm}, d_{2 \mathrm{u}}=d_{4 \mathrm{u}}=$ $3.94 \mathrm{~mm}, d_{5 \mathrm{u}}=5 \mathrm{~mm}, d_{1 \mathrm{v}}=d_{5 \mathrm{v}}=3.57 \mathrm{~mm}, d_{2 \mathrm{v}}=d_{4 \mathrm{v}}=4.61 \mathrm{~mm}$, $d_{5 \mathrm{v}}=5.87 \mathrm{~mm}, w_{1 \mathrm{u}}=w_{2 \mathrm{u}}=w_{3 \mathrm{u}}=w_{4 \mathrm{u}}=w_{5 \mathrm{u}}=w_{1 \mathrm{v}}=w_{2 \mathrm{v}}=w_{3 \mathrm{v}}=$ $w_{4 \mathrm{v}}=w_{5 \mathrm{v}}=0.5 \mathrm{~mm}$.

\section{REFERENCES}

[1] T. Ebbesen, H. Lezec, H. Ghaemi, T. Thio, and P. A. Wolff, "Extraordinary optical transmission through sub-wavelength hole arrays," Nature 391, 667-669 (1998). https://doi.org/10.1038/35570

[2] S. Guenneau, S. Anantha Ramakrishna, S. Enoch, Sangeeta Chakrabarti, G. Tayeb, B. Gralak, "Cloaking and imaging effects in plasmonic checkerboards of negative $\varepsilon$ and $\mu$ and dielectric photonic crystal checkerboards," Photonics and Nanostructures - Fundamentals and Applications, Vol. 5, no 2-3, pp. 63-73, 2007

[3] S. I. Grosz, D. C. Skigin, and A. N. Fantino, "Resonant effects in compound diffraction gratings: Influence of the geometrical parameters of the surface," Phys Rev E Stat Nonlin Soft Matter Phys. 2002 May;65(5 Pt 2):056619. doi: 10.1103/PhysRevE.65.056619. Epub 2002 May 22. PMID: 12059743.

[4] Y. Jiang, L. Wang, J. Wang, C. Nwakanma Akwuruoha, and W. Cao, "Ultra-wideband high-efficiency reflective linear-to-circular polarisation converter based on metasurface at terahertz frequencies," Opt. Express, vol. 25, pp. 27616-27623, 2017

[5] D. C. Skigin and R. A. Depine, "Transmission resonances of metallic compound gratings with subwavelength slits," Phys. Rev. Lett., vol. 95, Nov. 2005, Art. no. 217402

[6] A. P. Hibbins, I. R. Hooper, M. I. Lockyear, and J. R. Sambles, "Microwave transmission of a compound gratings," Phys. Rev. Lett., vol. 96, Jun. 2006, Art. no. 257402

[7] D. C. Skigin, V. V. Veremey and R. Mittra, "Superdirective radiation from finite gratings of rectangular grooves," in IEEE Transactions on Antennas and Propagation, vol. 47, no. 2, pp. 376-383, Feb. 1999, doi: $10.1109 / 8.761078$

[8] F. Medina, F. Mesa and D. C. Skigin, "Extraordinary Transmission Through Arrays of Slits: A Circuit Theory Model," in IEEE Transactions on Microwave Theory and Techniques, vol. 58, no. 1, pp. 105-115, Jan. 2010, doi: 10.1109/TMTT.2009.2036341.

[9] C. Molero, R. Rodríguez-Berral, F. Mesa and F. Medina, "Dynamical Equivalent Circuit for 1-D Periodic Compound Gratings," in IEEE Transactions on Microwave Theory and Techniques, vol. 64, no. 4, pp. 1195-1208, April 2016, doi: 10.1109/TMTT.2016.2531663.

[10] A. K. Rashid, B. Li and Z. Shen, "An overview of threedimensional frequency-selective structures," in IEEE Antennas and Propagation Magazine, vol. 56, no. 3, pp. 43-67, June 2014, doi: 10.1109/MAP.2014.6867682.

[11] W. Tang, J. Zhu, C. Wang, J. Ge, Z. Yu and W. Zhuang, "Waveguide 3-D FSSs by 3-D printing technique," 2016 International Conference on Electromagnetics in Advanced Applications (ICEAA), 2016, pp. 675678

[12] C. Molero Jimenez, E. Menargues and M. García-Vigueras, "All-Metal 3-D Frequency-Selective Surface With Versatile Dual-Band Polarisation Conversion," in IEEE Transactions on Antennas and Propagation, vol. 68, no. 7, pp. 5431-5441, July 2020, doi: 10.1109/TAP.2020.2975270.

[13] A. A. Omar and Z. Shen, "Thin 3-D Bandpass Frequency-Selective Structure Based on Folded Substrate for Conformal Radome Applications," in IEEE Transactions on Antennas and Propagation, vol. 67, no. 1, pp. 282-290, Jan. 2019, doi: 10.1109/TAP.2018.2876706.

[14] A. A. Omar, J. Kim and W. Hong, "A 3-D Lumped-ComponentsFree Absorptive Frequency-Selective Transmission Structure Featuring Very Wide Two-Sided Absorption Bandwidths," in IEEE Antennas and Wireless Propagation Letters, vol. 19, no. 5, pp. 761-765, May 2020, doi: 10.1109/LAWP.2020.2979351

[15] A. Sadeqi, H. Rezaei-Nejad, R. E. Owyeung, and S. Sonkusale "Three dimensional printing of metamaterial embedded geometrical optics (MEGO)," Microsyst Nanoeng vol. 5, no. 16, 2019, https://doi.org/10.1038/s41378-019-0053-6

[16] M., Harnois, M. Himdi, W. Yong, S. Abdul-Rahim, K. Tekkouk, and N. Cheval, "An Improved 1072 Fabrication Technique for the 3-D Frequency Selective Surface based on Water Transfer Printing Technology," Scientific Reports 2020, 10, 1714. doi:10.1038/s41598-020-58657-5.

[17] X. Huang, C. Yang, Z. Lu, and P. Liu, "A novel frequency selective structure with quasi-elliptic bandpass response," in IEEE Antennas Wireless. Propag. Lett., vol. 11, pp. 1497-1500, 2012.

[18] C. Molero and M. García-Vigueras, "Circuit Modeling of 3-D Cells to Design Versatile Full-Metal Polarizers," in IEEE Transactions on Microwave Theory and Techniques, vol. 67, no. 4, pp. 1357-1369, April 2019, doi: 10.1109/TMTT.2019.2898828.

[19] N. Chahat, B. Cook, H. Lim and P. Estabrook, "All-Metal DualFrequency RHCP High-Gain Antenna for a Potential Europa Lander,' in IEEE Transactions on Antennas and Propagation, vol. 66, no. 12, pp. 6791-6798, Dec. 2018

[20] R. Rodríguez-Berral, F. Mesa and F. Medina, "Analytical Multimodal Network Approach for 2-D Arrays of Planar Patches/Apertures Embedded in a Layered Medium," in IEEE Transactions on Antennas and Propagation, vol. 63, no. 5, pp. 1969-1984, May 2015, doi: 10.1109/TAP.2015.2406885

[21] A. Alex-Amor, F. Mesa, Á. Palomares-Caballero, C. Molero and P. Padilla, "Exploring the Potential of the Multi-Modal Equivalent Circuit Approach for Stacks of 2-D Aperture Arrays," in IEEE Transactions on Antennas and Propagation, vol. 69, no. 10, pp. 6453-6467, Oct. 2021, doi: 10.1109/TAP.2021.3070150

[22] D. M. Pozar, "Microwave Engineering," Fourth edition, Wiley, 2011. New York, 2012

[23] S. -Y. Wang, J. -D. Bi, W. Liu, W. Geyi and S. Gao, "PolarizationInsensitive Cross-Polarization Converter,' in IEEE Transactions on Antennas and Propagation, vol. 69, no. 8, pp. 4670-4680, Aug. 2021, doi: 10.1109/TAP.2021.3060087.

[24] A. A. Omar, Z. Shen and H. Huang, "Absorptive Frequency-Selective Reflection and Transmission Structures," in IEEE Transactions on Antennas and Propagation, vol. 65, no. 11, pp. 6173-6178, Nov. 2017, doi: 10.1109/TAP.2017.2754463. 